\title{
Aportaciones al conocimiento de la flora vascular de las montañas de Sanabria (Zamora, NO de España)
}

\author{
Patricio Bariego Hernández ${ }^{1}$
}

\begin{abstract}
Resumen: Bariego, P. 2011. Aportaciones al conocimiento de la flora vascular de las montañas de Sanabria (Zamora, NO de España). Bot. Complut. 35: 49-58.

Se señalan y comentan 49 táxones de interés corológico, biogeográfico o conservacionista en la comarca de Sanabria, un área montañosa de notable interés florístico en el NO de Zamora (España). Todos ellos están escasamente citados en este territorio y algunos son novedad para la provincia de Zamora como Anemone trifolia subsp. albida, Ranunculus carlittensis o Rubus saxatilis.
\end{abstract}

Palabras clave: corología, plantas vasculares, Sierra Segundera, Sierra de la Cabrera, Sanabria, Zamora, Orense, España.<

Contents: Bariego, P. 2011. Contribution to the knowledge of the vascular flora of the mountain range of Sanabria (province of Zamora, NW Spain). Bot. Complut. 35: 49-58.

Fortynine taxa of chorological, biogeographical or conservation interest in the mountain range of Sanabria, a floristic hot-spot in the NW of Zamora province (Spain), are remarked and commented. All of them are poorly referenced there and some of them are cited in the Zamora province for the first time like Anemone trifolia subsp. albida, Ranunculus carlittensis or Rubus saxatilis.

Key words: chorology, vascular flora, Sierra Segundera, Sierra de la Cabrera, Zamora province, Orense province, Spain.

\section{INTRODUCCIÓN}

A lo largo de los últimos años se ha venido realizando un intenso trabajo de prospección de la flora vascular de la comarca de Sanabria, una comarca de alto valor natural en el noroccidente de Zamora que incluye las montañas que conforman la Sierra Segundera y una parte de la Sierra de la Cabrera. Este trabajo ha servido para completar el conocimiento de la flora vascular de este territorio de notable interés florístico que anteriores trabajos monográficos habían acometido (Losa 1949, Losa 1950, García López et al. 1992), así como el realizado en las vertientes leonesas y orensanas contiguas (Nieto Feliner 1985, Ortiz 1986b).

En la presente nota se recogen nuevos datos para un conjunto de plantas vasculares cuya presencia en este amplio territorio no se conocía con anterioridad (se señalan con * las que además constituyen novedad provincial para Zamora) y otras que presentan cierto interés desde el punto de vista biogeográfico o conservacionista.

Para cada taxón, ordenados según el criterio de Flora iberica 0 , en el caso de las familias no publicadas en esta obra, de Flora Europaea, se indican los datos referentes a su localización geográfica (provincia, municipio, toponímia, UTM 1x1 y altitud), el hábitat donde ha sido observado, la fecha y autor/es de la recolección y un número de identificación del colector P. Bariego (PB). Todos los pliegos han sido depositados en el herbario de la Universidad de Salamanca (SALA). Además se comentan brevemente algunos datos referidos a su distribución geográfica, ecología o estado de conservación en este territorio.

\section{RESULTADOS}

\section{Osmunda regalis $\mathrm{L}$.}

ESPAÑA: ORENSE: Viana do Bolo, Embalse de San Sebastián, 29TPG6666, $1080 \mathrm{~m}$, paredes rezumantes, 11-VII-2004, P. Bariego, PB 3543. ZamoRa: Galende, Ribadelago, Cañón del Tera, 29TPG8567, $1130 \mathrm{~m}$, orilla del Tera, 30-V- 2002, P. Bariego, PB 1137.

Novedad para el macizo de la Sierra Segundera, donde aparece restringido a las zonas más térmicas. Recien-

${ }^{1}$ C/ Candelaria Ruiz del Árbol, 37, $4^{\circ}$ A, E-49017 Zamora, España.pbariego@hotmail.es

Recibido: 11 abril 2011. Aceptado: 9 mayo 2011 
temente completabamos la distribución de este helecho en la provincia de Zamora (Bariego et al. 2004: 58).

\section{Dryopteris expansa (K. Presl) Fraser-Jenkins \& Jermy}

ESPAÑA: ZamoRa: Porto, Circo de la Freixiña, 29TPG8074, $1840 \mathrm{~m}$, canchales húmedos, 20-VIII-2002, P. Bariego, PB 990; Porto, pr. Laguna de Lacillo, 29TPG8274, $1720 \mathrm{~m}$, canchal gneísico, 27VII-2002, E. Rico \& P. Bariego, PB 843; Porto, Circo de Peña Negra, 29TPG8279, $1890 \mathrm{~m}$, canchal, 16-VIII-2002, P. Bariego, PB 862.

Restringido a zonas de canchal a elevada altitud, se trata de un pteridófito que ya había sido citado en la Sierra Segundera (Aedo et al. 1993: 351). Completamos ahora la distribución de este raro helecho en este ámbito, aportando nuevas localidades.

\section{Trollius europaeus L.}

ESPAÑA: Zamora: Porto, Sierra de los Hoyos, 29TPG7874, 1650 $\mathrm{m}$, herbazales megaforbios, 12-VII-2003, P. Bariego, PB 3392; Porto, Circo de la Surbia, 29TPG8178, $1870 \mathrm{~m}$, repisas umbrosas y frescas en roquedos, 19-VII-2002, P. Bariego, PB 1229; Porto, entre Vega de Tera y Vega de Conde, 29TPG8273, $1560 \mathrm{~m}$, bordes de turbera, 19-VII2002, P. Bariego, PB 1068.

Tras la referencia de Losa (1949: 76), única mención provincial conocida, no había vuelto a ser citado en las montañas sanabresas. Es una planta poco frecuente en estas sierras, que participa en comunidades de megaforbios y otros herbazales higrófilos en enclaves más o menos frescos y en repisas de roquedo protegidas a elevada altitud.

\section{Actaea spicata L.}

ESPAÑA: ZAMORa: Porto, 29TPG8164, $1600 \mathrm{~m}$, canchales húmedos, 18-VII-2002, P. Bariego, PB 1060; Cobreros, Quintana de Sanabria, pr. Cascada de Sotillo, 29TPG8562, $1400 \mathrm{~m}$, bosquete ripario, 24-V-2002, P. Bariego, PB 1007.

Taxon que aparece disperso por la Sierra Segundera, participando en comunidades megafórbicas tanto en bosques frescos como en roquedos y canchales húmedos. Añadimos nuevas localidades a las señaladas por Losa (1950: 498) y Casaseca et al. (1990: 116).

\section{*Anemone trifolia subsp. albida (Mariz) Ulbr.}

ESPAÑA:ZAmora: Pías, Villanueva de la Sierra, Valle del Bibey, 29TPG6360, 1340 m, abedular, 20-VIII-2002, P. Bariego, PB 1882; Pí- as, 29TPG6460, $1180 \mathrm{~m}$, robledal mixto, 19-V-2003, P. Bariego PB 3332; Pías, 29TPG6662, 1060 m, aliseda, 19-V-2003, P. Bariego, PB 3338.

Endemismo atlántico que solo era conocido de Galicia y el norte de Portugal (Silva Pando 2008: 35). Se trata de una destacada novedad para la flora de Zamora y de la Comunidad de Castilla y León. En este ámbito vegeta en claros de abedular y robledal, donde podría convivir con Anemone nemorosa L. que ha sido citada en las inmediaciones (Aedo et al. 1993: 355).

\section{*Ranunculus carlittensis (Sennen) Grau}

ESPAÑA: ZAMORA: Porto, Valdesirgas, 29TPG7468, $1440 \mathrm{~m}$, acebeda, 19-V-2003, P. Bariego, PB 3328.

Novedad florística para el macizo Galaico-Leonés y para la flora zamorana. Esta microespecie incluida en el grupo auricomus presenta una peculiar distribución ibérica (Pirineos orientales, Montes de Soria, León y Álava), por lo que la cita que aportamos constituiría una nueva aportación al conocimiento de sus poblaciones occidentales. Se trata de un complejo grupo, no exento de ciertas dificultades taxonómicas (Cook et al. 1986), por lo que parecería adecuado realizar un detallado estudio de estas poblaciones. En este territorio tan sólo ha sido localizada una población en el seno de la interesante acebeda de Valdesirgas.

\section{Ranunculus amplexicaulis $\mathrm{L}$.}

ESPAÑA: Zamora: Galende, San Martín de Castañeda, Gencianal, 29TPG8670, $1720 \mathrm{~m}$, pastizales psicroxerófilos, 09-VI-2002, $P$. Bariego, PB 1036.

Añadimos una nueva localidad a la previamente señalada en estas montañas (Aedo et al. 1993: 355). Este ranúnculo de flor blanca aparece disperso en pastizales psicroxerófilos, en piornales de cumbre y en matorrales de brecina, sin llegar a ser una planta frecuente en estas montañas.

\section{*Ranunculus parnassiifolius subsp. cabrerensis Rothm.}

ESPAÑA: ZamoRA: Porto, pr. Laguna de Patos, 29TPG8578, 2040 m, gleras finas de pizarras, 30-VII-2002, P. Bariego, PB 848; Porto, pr. Laguna de Patos, hacia el Picón, 29TPG8578, 2010 m, gleras finas de pizarras, 30-VII-2002, P. Bariego, PB 2402; San Justo, La Plana, 29TPG8876, $2000 \mathrm{~m}$, gleras finas de pizarras, 20-VI-2002, P. Bariego \& A. Gallego, PB 1152 
A pesar de que su presencia en este conjunto montañoso ya era conocida, dado que la descripción de la subespecie se realizó con material del cercano Picón de La Baña (León) (Nieto Feliner 1985: 45), aún no había sido citado en el ámbito administrativo zamorano. Se aportan, por tanto, las localidades conocidas de este interesante endemismo que vive estrechamente ligado a gleras finas de pizarras cubiertas de nieve hasta la primavera en la zona de ruptura de pendiente de las cabeceras de los circos glaciares de la Sierra de la Cabrera.

\section{Thalictrum minus L. subsp. minus}

ESPAÑA: ORENSE: Pradorramisquedo, pr. Central de San Agustín, 29TPG6667, $1100 \mathrm{~m}$, orlas de rebollar, 22-VI-2003, P. Bariego, PB 2880. Zamora: Pías, 29TPG6662, $1060 \mathrm{~m}$, aliseda, 14-VI-2003, P. Bariego, PB 2875; Porto, arroyo de Valdeinfierno, 29TPG7669, $1440 \mathrm{~m}$, bordes del arroyo, 11-VII-2003, P. Bariego, PB 3383; Porto, 29TPG7570, $1260 \mathrm{~m}$, herbazales en el río Bibey, 22-VI-2003, P. Bariego, PB 2872.

Segunda cita provincial zamorana, a añadir a la del extremo sudoccidental de la provincia (Sánchez Rodríguez 1986: 373). Las localidades que aportamos amplían su área provincial y constituyen novedad para la flora de la Sierra Segundera.

\section{Corydalis cava (L.) Schweigger \& Koerte subsp. cava}

ESPAÑA: ZAMora: Porto, Valdesirgas, 29TPG7468, $1440 \mathrm{~m}$, acebeda, 19-V-2003, P. Bariego, PB 3327; Galende, San Martín de Castañeda, Cueva de San Martín, 29TPG8470, 1380 m, acebeda, 22-V-2002, P. Bariego, PB 774 .

Nuevas localidades a añadir a las indicadas por Cabezas et al. (1987: 96) y Casaseca et al. (1990: 116), únicas conocidas en la provincia de Zamora, a la de Ortiz (1986a: 192) de la vertiente orensana de Trevinca y a las de Gómez Vigide et al. (2006: 55) en el tramo orensano del valle alto del Bibey. Completamos la distribución de este geófito poco frecuente y estrechamente ligado a los bosques más frescos y umbrosos de este área.

\section{*Sagina saginoides (L.) Karsten}

ESPAÑA: Zamora: Porto, pr. Fuente Los Tejos, 29TPG8268, $1700 \mathrm{~m}$, brezal de turbera, 09-VIII-2002, P. Bariego, A. Gallego \& M. Santos, PB 2453; Galende, San Martín de Castañeda, pr. Laguna de Peces, 29TPG8671, $1660 \mathrm{~m}$, turbera, 27-VI-2002, P. Bariego \& A. Gastón, PB 1191; Galende, San Martín de Castañeda, Covadosos,
29TPG8670, 1630 m, turbera, 28-VII-2002, P. Bariego, M. Santos \& L. Delgado, PB 2368.

Novedad para la flora vascular sanabresa y de la provincia de Zamora. Se trata de una especie estrechamente ligada a los arroyos nacientes y zonas encharcadas dentro de turberas de las zonas altas de la Sierra Segundera.

\section{Rumex suffruticosus Gay ex Willk.}

ESPAÑA: Zamora: Porto, Entre Riopedro y Vidulante, 29TPG8678, 1970 m, glera, 30-VII-2002, P. Bariego, PB 2404; Porto, Riopedro, 29TPG8777, $2000 \mathrm{~m}$, gleras finas, 30-VII-2002, P. Bariego, PB 812; Galende, entre Vega de Tera y Moncalvo, 29TPG8074, 1860 m, gleras finas móviles, 22-VIII-2002, P. Bariego, PB 992; Porto, pr. Laguna de Patos, 29TPG8578, $2000 \mathrm{~m}$, afloramiento de pizarras, 30VII-2002, P. Bariego, PB 847

Segunda cita provincial para este endemismo que ya había sido localizado en las cumbres de la Sierra de la Cabrera, tanto en la vertiente zamorana como leonesa (García Río \& Navarro 1991: 149; Nieto Feliner 1985: 70). Es un caméfito relativamente frecuente en las gleras de las zonas elevadas de las Sierras Segundera y Cabrera.

\section{Rumex longifolius DC.}

ESPAÑA: ZaMORA: Porto, Valdecasares, 29TPG7961, $1850 \mathrm{~m}$, neveros, 08-VII-2003, P. Bariego, PB 3405.

Nueva localidad a añadir a la previamente conocida en la Segundera (Aedo et al. 1994: 69). En estas Sierras es una planta muy escasa que se localiza en entornos ruderales a elevada altitud.

\section{Viola pyrenaica Ramond ex DC.}

ESPAÑA: Zamora: Galende, Ribadelago, Cañón del Tera, 29TPG8568, 1200 m, rebollar, 30-V-2002, P. Bariego, PB 1142.

Aportamos una nueva localidad a la ya existente en zonas basales de la comarca sanabresa (Aedo et al. 1993: 358). Es un taxón poco frecuente en este área, ligado a zonas algo ruderales en claros forestales.

\section{Viola parvula Tineo}

ESPAÑA: ZamoRa: Galende, Ribadelago, cañada sanabresa, 29TPG8564, 1520 m, matorral de brecina, 06-VII-2002, P. Bariego, PB 
1133; Galende, San Martín de Castañeda, pr. Laguna de Peces, 29TPG8670, 1690 m, piornal de cumbre, 27-VI-2002, P. Bariego, PB 2205.

Aportamos dos nuevas localidades a la ya existente en la Sierra Segundera (Aedo et al., 1993: 358). Este delicado terófito crece en suelos arenosos y algo alterados al abrigo de diferentes tipos de matorral.

\section{Sesamoides minor (Lange) Kuntze}

ESPAÑA: Zamora: Porto, entre Peña Negra y PeñaTrevinca, 29TPG8179, $2040 \mathrm{~m}$, brezal con aulaga sanabresa, 16-VIII-2002, $P$. Bariego, PB 864; Galende, San Martín de Castañeda, Peña Cabrita, 29TPG8672, $1880 \mathrm{~m}$, brezal bajo con aulaga sanabresa, 29-VII-2002, P. Bariego, PB 853; Galende, San Martín de Castañeda, Peña Cabrita, 29TPG8672, $1880 \mathrm{~m}$, brezal bajo con aulaga sanabresa, 30-VII-2002, P. Bariego, PB 811; Trefacio, Bulante, 29TPG8775, $1980 \mathrm{~m}$, comunidades quionófilas en nevero, 29-VI-2004, P. Bariego, A. Gallego, L. P. Gavilán, H. Sainz, E. Blanco \& J. Díez, PB 2608; Galende, San Martín de Castañeda, pr. Laguna de Peces, 29TPG8772, 1700 m, escobonal, 27-VI-2002, P. Bariego \& A. Gastón, PB 1194; Trefacio, Murias, Pinar de Murias, 29TPG9170, $1700 \mathrm{~m}$, brezal con carquesas, 03-VII2002, P. Bariego, PB 969.

Endemismo del macizo galaico-leonés ya citado en el macizo de Trevinca (Izco \& Ortiz 1985: 61 y 62) y cuya presencia en el extremo oriental de la Cabrera zamorana ha sido recientemente denunciada por Martín Bravo \& Luceño (2007: 226). No es rara en suelos desnudos en zonas de nevero y en claros de los matorrales almohadillados de las zonas elevadas de la Sierra.

\section{Pyrola minor $\mathrm{L}$.}

ESPAÑA: Zamora: Pías, Villanueva de la Sierra, Valle del Bibey, 29TPG6360, $1340 \mathrm{~m}$, abedular, 20-VIII-2002, P. Bariego, PB 1887; Requejo, Tejedelo, 29TPG8254, $1420 \mathrm{~m}$, enclave cenagoso en bosquete mixto con abedules y tejos, 17-VIII-2002, P. Bariego, PB 1853; Cobreros, Sotillo de Sanabria, por encima de la Cascada de Sotillo, 29TPG8561, 1500 m, abedular, 08-VIII-2002, P. Bariego, A. Gallego \& M. Santos, PB 2452; Galende, Ribadelago, cañón del Segundera, 29TPG8464, 1370 m, acebeda, 08-VI-2002, P. Bariego, PB 1204; Cobreros, Sotillo de Sanabria, Valle de Sotillo, 29TPG8761, 1200 m, abedular, 01-VI-2002, P. Bariego, PB 2061; Galende, Vega de Tera, 29TPG8371, $1560 \mathrm{~m}$, prebosque en arroyo, 02-VII-2002, P. Bariego, PB 2237.

Añadimos nuevas localidades a las ya conocidas de la cabecera de los valles del río Bibey y Truchas (Casaseca et al. 1990: 118) y de la vertiente orensana de Trevinca (Ortiz 1986a: 193), completando su distribución en la Sierra Segundera. Se trata de una planta poco frecuente en este territorio, ligada a zonas encharcadas y rezumantes en el seno de bosques densos y frescos como abedulares $\mathrm{y}$ acebedas.

\section{*Primula elatior subsp. intricata (Gren. \& Godron) Widmer}

ESPAÑA: Zamora: Porto, Circo de la Surbia, 29TPG8178, 1820 $\mathrm{m}$, herbazales en repisas umbrosas de roquedos, 19-VII-2002, P. Bariego, PB 814; Porto, Circo de la Surbia, 29TPG8178, 1870 m, repisas umbrosas y frescas en roquedos, 19-VII-2002, P. Bariego, PB 1228; Galende, San Martín de Castañeda, Cueva de San Martín, 29TPG8470, $1380 \mathrm{~m}$, acebeda, 22-V-2002, P. Bariego, PB 768.

Interesante novedad para la provincia de Zamora, aunque la imprecisa cita de Losa (1949: 106, sub. P. officinalis Jacq.) en Puebla de Sanabria podría corresponder a este taxon. En cualquier caso, se trata de una planta muy escasa en esta zona, que crece en pastos rezumantes en repisas de roquedo y en el seno de bosques frescos como acebedas y abedulares.

Umbilicus heylandianus Webb \& Berth.

ESPAÑA: OREnSE: Viana do Bolo, Pradorramisquedo, pr. Central de San Agustín, 29TPG6667, 1100 m, orlas de rebollar, 22-VI-2003, P. Bariego, PB 2876.

Novedad para la flora vascular de la Sierra Segundera y segunda cita provincial para Orense (Pulgar et al. 1998: 94). Crece en herbazales de orla forestal soleados.

\section{*Sempervivum vicentei Pau}

ESPAÑA: Zamora: Porto, Circo de la Surbia, 29TPG8178, 1820 $\mathrm{m}$, herbazales en repisas umbrosas de roquedos, 19-VII-2002, $P$. Bariego, PB 813; Porto, Peña Negra, 29TPG8280, 2110 m, roquedos, 16VIII-2002, P. Bariego, PB 819.

Concretamos diferentes localidades en el ámbito zamorano del macizo de Trevinca para este taxon que, a pesar de las referencias previas en dicho macizo (Izco \& Ortiz 1985: 59), únicamente poseía en territorio zamorano la referencia provincial de Flora iberica (Roselló, 1997). Es una planta relativamente frecuente en espolones rocosos de las zonas de cumbre más elevadas.

\section{*Sedum anglicum Hudson}

ESPAÑA:ZAMORA: Porto, Valdecasares, 29TPG7961, $1850 \mathrm{~m}$, neveros, 08-VII-2003, P. Bariego, PB 3407; Trefacio, pr. Laguna de Yeguas 
o Cubillas, 29TPG8873, $1820 \mathrm{~m}$, repisas en roquedos, 20-VI-2002, P. Bariego \& Gallego, PB 957; Porto, Circo de Peña Negra, 29TPG8279, 1900 $\mathrm{m}$, roquedos umbrosos, 16-VIII-2002, P. Bariego, PB 2458; Trefacio, Sierra de Trefacio, 29TPG8874, $1885 \mathrm{~m}$, repisas en espolones rocosos, 29VII-2002, P. Bariego, PB 851; Porto, pr. Laguna de Lacillo, 29TPG8274, $1700 \mathrm{~m}$, cervunal, 27-VII-2002, E. Rico \& P. Bariego, PB 824.

Aportamos numerosas localidades de este taxon nuevo en la provincia de Zamora que, a juzgar por su relativa frecuencia en esta área, quizás haya pasado desapercibido por confusión con otros táxones del género. Crece en repisas rocosas y suelos desnudos sometidos a procesos de crioturbación en las zonas altas de la Sierra.

\section{*Sedum candollei Raym.-Hamet}

ESPAÑA: ZAMORA: Porto, Valdecasares, 29TPG7861, $1810 \mathrm{~m}$, comunidades quionófilas en nevero, 27-VII-2002, E. Rico \& P. Bariego, PB 844; Trefacio, Tres Cruces, 29TPG8775, 1990 m, nevero, 30VII-2002, P. Bariego, PB 2391; Porto, pr. Moncalvillo, 29TPG8073, $1990 \mathrm{~m}$, pastizales pioneros, 27-VI-2002, P. Bariego \& A. Gastón, PB 1105; Porto, Moncalvo, 29TPG8074, $1980 \mathrm{~m}$, nevero, 27-VII-2002, P. Bariego \& E. Rico, PB 2364.

Aportamos diferentes localidades en la vertiente zamorana en la Sierra Segundera para este taxon que ya era conocido en el Macizo de Trevinca (Nieto Feliner, 1985: 94, sub. Mucizonia sedoides (DC) D. A. Webb). A pesar de ello, la única referencia provincial zamorana conocida era la de Flora iberica (Castroviejo et al., 1997). Es una planta de llamativa floración que crece en suelos descarnados sometidos a procesos de crioturbación en enclaves de nevero.

\section{Saxifraga stellaris L.}

ESPAÑA: Zamora: Porto, Moncalvo, 29TPG8174, $2000 \mathrm{~m}$, herbazales higrófilos en zona de acumulación de nieve, 27-VI-2002, $P$. Bariego \& A. Gastón, PB 1073; Trefacio, 29TPG8775,1980 m, naciente, 21-VI-2002, P. Bariego \& A. Gallego, PB 2154.

Nuevas localidades de este taxon, ligado a nacientes y ambientes fontinales de alta montaña, a añadir a las que aporta Losa (1949: 84), únicas conocidas en la provincia de Zamora.

\section{*Rubus saxatilis L.}

ESPAÑA: Zamora: Cobreros, Sotillo de Sanabria, por encima de la Cascada de Sotillo, 29TPG8561, 1500 m, abedular, 08-VIII-2002, P. Bariego, A. Gallego \& M. Santos, PB 2451.
Novedad provincial destacada ya que se trata de una planta rara en la Península Ibérica, donde se restringe a escasas localidades en la Cordillera Cantábrica, Pirineos y el Sistema Ibérico (Gómez Serrano \& Mayoral 2006: 32). La referencia más cercana se sitúa en el entorno de Valporquero (León) (Carbó et al. 1977: 78). La población de la Sierra Segundera, límite occidental de su área de distribución ibérica, forma un tapiz en el sotobosque de un abedular fresco.

\section{*Potentilla pyrenaica Ramond ex DC.}

ESPAÑA: Zamora: Galende, Valle de Tejos, 29TPG8369, 1630 $\mathrm{m}$, herbazales en pie de cantil, 13-VIII-2002, P. Bariego, PB 925; Cobreros, Sotillo de Sanabria, pr. Cascada de Sotillo, 29TPG8561, 1500 $\mathrm{m}$, orlas deabedular, 08-VIII-2002, M. Santos, A. Gallego \& P. Bariego, PB 881; Porto, Circo de Lacillo, 29TPG8174, $1860 \mathrm{~m}$, herbazales megaforbios, 27-VII-2002, E. Rico \& P. Bariego, PB 832.

Novedad provincial, si bien ya ha sido citada de zonas próximas en el ámbito orensano del valle alto del Bibey (Gómez Vigide et al. 2005: 59). En Sanabria aparece ligada a herbazales megafórbicos, frecuentemente en orlas forestales y otros enclaves umbrosos como cantiles 0 roquedos.

\section{Circaea lutetiana L. subsp. lutetiana}

ESPAÑA: Zamora: Galende, San Martín de Castañeda, Playa de la Sirena, 29TPG8866, $1000 \mathrm{~m}$, aliseda, 10-VIII-2002, P. Bariego, PB 872; Galende, Ribadelago, Seoane, 29TPG8766, 1010 m, roquedos umbrosos, 26-VI-2002, P. Bariego \& K. Marhold, PB 1122; Galende, Ribadelago, Seoane, 29TPG8766, $1010 \mathrm{~m}$, roquedos umbrosos, 19-VIII2002, P. Bariego, PB 879.

Nuevas localidades de este taxon a añadir a la mención de García López et al. (1992: 71), única conocida en la provincia. Es una planta muy escasa en estas montañas, que crece en localizaciones umbrosas en el seno de bosques frescos.

\section{*Euphorbia hyberna L. subsp. hyberna}

ESPAÑA: Zamora: Porto, pr. embalse de San Sebastián, 29TPG7268, 1240 m, rebollar, 18-V-2002, P. Bariego 726; Pías, 29TPG6662, $1060 \mathrm{~m}$, aliseda, 19-V-2003, P. Bariego 3335.

Novedad provincial, aunque no lo es para el valle del Bibey donde ya la herborizó Merino en el ámbito administrativo orensano (Losa 1948: 369). 


\section{*Selinum pyrenaeum (L.) Gouan}

ESPAÑA: ZamoRa: Porto, pr. embalse de Playa, 29TPG8065, $1600 \mathrm{~m}$, cervunal, 18-VI-2002, P. Bariego, PB 1062; Porto, Las Tres Marías, 29TPG8275, $1850 \mathrm{~m}$, pastizal psicroxerófilo, 05-VIII-2002, $P$. Bariego \& M. Santos, PB 2430; Porto, Circo de Peña Negra, 29TPG8279, 1890 m, cervunal, 16-VIII-2002, P. Bariego, PB 863; Porto, pr. Laguna de Lacillo, 29TPG8274, $1700 \mathrm{~m}$, cervunal, 27-VII-2002, E. Rico \& P. Bariego, PB 827; Porto, Valle alto del Tera, 29TPG8377, $1640 \mathrm{~m}$, cervunal, 19-VII-2002, P. Bariego, PB 1219.

Aportamos diferentes localidades en la vertiente zamorana de la Sierra Segundera de este taxon cuya única referencia provincial previa es la de Flora iberica (Gómez 2003). Habita en cervunales y otros pastos húmedos de las zonas elevadas de la Sierra.

\section{${ }^{*}$ Ligusticum lucidum Mill.}

ESPAÑA: Zamora: Galende, Puente de Sanabria, ribera del Tera, 29TPG9561, 920 m, aliseda, 26-VII-2002, P. Bariego, PB 796.

Novedad provincial, aunque ya había sido citado en la vertiente leonesa de estos macizos montañosos (Nieto Feliner 1985: 128). Únicamente se ha localizado creciendo en claros de aliseda en cascajares fluviales del río Tera.

\section{Ferulago capillaris (Link ex Sprengel) Coutinho}

ESPAÑA: Zamora: Galende, Cañón del Tera, 29TPG8668, 1500 $\mathrm{m}$, rebollar arbustivo, 25-VI-2002, P. Bariego, PB 971; Galende, San Martín de Castañeda, 29TPG8868, 1520 m, escobonal, 28-VII-2002, L. Delgado, M. Santos \& P. Bariego, PB 821; Galende, San Martín de Castañeda, Corona, 29TPG8767, $1420 \mathrm{~m}$, orlas de rebollar, 02-VIII2002, P. Bariego, PB 793.

Novedad para la flora de la Sierra Segundera zamorana, aportamos a las localidades a añadir a las ya conocidas en otro ámbito provincial (García Río \& Navarro 1994: 70). Se trata de un megaforbio que crece en el seno de matorrales y en el entorno de bosques poco maduros o ligeramente degradados.

\section{*Myosotis stolonifera (DC.) Gay ex Leresche \& Levier}

ESPAÑA: ZAMora: Cobreros, pr. Laguna de Sotillo, 29TPG8463, 1610 m, turbera, 08-VII-2002, PB 2289; Galende, San Martín de Castañeda, Covadosos, 29TPG8671, $1640 \mathrm{~m}$, turbera, 11-VI-2002, P. Bariego, PB 1048.

Novedad provincial. Es una planta distribuida por las montañas del cuadrante noroccidental ibérico, conocida en la vertiente leonesa de este macizo montañoso (Valdés 2008: 105). En este territorio se restringe a turberas y nacientes en las zonas altas.

\section{Ajuga reptans $\mathrm{L}$.}

ESPAÑA: Zamora: Pías, 29TPG6662, $1060 \mathrm{~m}$, aliseda, 19-V2003, P. Bariego, PB 3336; Puebla de Sanabria, ribera del Castro, 29TPG9459, $900 \mathrm{~m}$, aliseda con abedules, 12-V-2002, P. Bariego \& A. Gallego, PB 914; Galende, Pedrazales, ribera del Tera, 29TPG9165, 990 m, aliseda, 21-V-2002, P. Bariego, PB 758.

Nuevas referencias a añadir a la única conocida en el ámbito provincial (Cabezas et al. 1987: 97). En este territorio las principales poblaciones están ligadas a alisedas, aunque puede crecer en otros enclaves húmedos y sombríos.

\section{*Antirrhinum meonanthum Hoffmans. \& Link}

ESPAÑA: ZAMORA: Porto, carretera a Orense, 29TPG7169, 1420 m, comunidades ruderales en cuneta, 31-VII-2003, P. Bariego, PB 3422; Porto, 29TPG7169, $1360 \mathrm{~m}$, cunetas con grava caliza, 15-VI-2003, $P$. Bariego, PB 2900.

Novedad provincial, aunque ya era conocida en la vertiente leonesa del macizo (Nieto Feliner 1985: 139). Su localización en una cuneta de carretera, en la que se han añadido áridos calcáreos, puede hacer sospechar sobre su origen antrópico en esta zona.

\section{Veronica nevadensis Pau}

ESPAÑA: ZaMora: Porto, pr. Moncalvillo, 29TPG8073, 1990 $\mathrm{m}$, turbera, 27-VI-2002, P. Bariego \& A. Gastón, PB 1107; Porto, embalse de Vega de Conde, 29TPG8475, $1600 \mathrm{~m}$, herbazales higrófilos em bordes de arroyo, 17-V-2002, P. Bariego, PB 756; Galende, San Martín de Castañeda, pr. Laguna de Peces, 29TPG8771, 1710 m, bordes de arroyos, 01-VI-2002, P. Bariego, PB 945.

Aportamos nuevas localidades a añadir a la señalada por Losa (1949: 112, sub. Veronica langei Lacaita). Es una planta relativamente frecuente en nacientes y ambientes fontinales de las zonas altas de la Sierra.

\section{*Euphrasia stricta D. Wollf ex J.F. Lehm}

ESPAÑA: Zamora: San Justo, Peñas de Bulante, 29TPG8776, $2000 \mathrm{~m}$, repisas umbrosas en roquedo, 30-VII-2002, P. Bariego, PB 2401. 
Conforme a la reciente revisión de Flora iberica (Vitek 2009), las plantas que hemos herborizado en la zonas elevadas de la Sierra Segundera corresponderían a este taxon. El material que sirve de soporte para esta cita, primera en la provincia, ha servido para la indicación provincial en Flora iberica.

\section{*Jasione crispa (Pourret) Samp. subsp. crispa}

ESPAÑA: Zamora: Rosinos de la Requejada, Escuredo, Sierra de la Cabrera, Vizcodillo, 29TQG0674, 1990 m, pastizales psicroxerófilos con Genista sanabriensis, 15-VIII-2001, P. Bariego, PB 84; Trefacio, Tres Cruces, 29TPG8775, $1990 \mathrm{~m}$, nevero, 30-VII-2002, P. Bariego, $\mathrm{PB} 2393$.

Confirmamos la presencia de este taxon en la vertiente zamorana de la Sierra Segundera, aunque en territorio leonés próximo ha sido citada con anterioridad (Nieto Feliner 1985: 150). Consideramos que es novedad provincial, atendiendo a que las referencias previas con material de las zonas bajas de estas Sierras corresponden a J. sessiliflora Boiss. \& Reuter. En este área ha sido localizada en enclaves puntuales formando parte de pastizales psicroxerófilos y matorrales almohadillados en zonas de acumulación de nieve y en lugares expuestos de las cumbres.

\section{*Omalotheca supina (L.) DC.}

ESPAÑA: Zamora: Porto, Moncalvo, 29TPG8074, $1980 \mathrm{~m}$, nevero, 27-VII-2002, P. Bariego \& E. Rico, PB 2362; Trefacio, Tres Cruces, 29TPG8775, 1990 m, nevero, 30-VII-2002, P. Bariego, PB 2392.

Aunque ya había sido citada en el Macizo de Trevinca (Laínz 1967: 39; Nieto Feliner 1985: 170), las citas que aportamos constituyen las primeras para la provincia de Zamora. Se localiza en zonas de ruptura de pendiente de la cumbre en la que se acumula nieve hasta bien entrada la primavera.

\section{*Antennaria dioica (L.) Gaertner}

ESPAÑA: Zamora: Porto, Valle alto del Tera, 29TPG8478, 2000 $\mathrm{m}$, brezal con carquesas, 14-VIII-2002, P. Bariego \& J. A. Sánchez Agudo, PB 2366; Porto, pr. Moncalvo, 29TPG8074, 2000 m, matorrales bajos con aulaga sanabresa, 27-VI-2002, P. Bariego \& A. Gastón, PB 1111.

Novedad provincial, aunque con referencias previas para el macizo Galaico-Leonés (Nieto Feliner 1985: 158). En estas montañas es una planta rara, localizada en pobla- ciones reducidas que forman parte de pastizales psicroxerófilos y matorrales almohadillados en lugares expuestos de las cumbres.

\section{*Phalacrocarpum oppositifolium (Brot.) Willk. subsp. oppositifolium}

ESPAÑA: Zamora: Trefacio, Bulante, 29TPG8775, 1980 m, comunidades quionófilas en nevero, 29-VI-2004, P. Bariego, A. Gallego, L. P. Gavilán, H. Sainz, E. Blanco \& J. Díez, PB 2609; Porto, Peñas de Bulante, 29TPG8776, $1960 \mathrm{~m}$, roquedos umbrosos, 20-VI-2002, P. Bariego \& A. Gallego, PB 1149; Robleda-Cervantes, San Juan de la Cuesta, Alto de San Juan, 29TPG9965, 1410 m, pizarras, cambrional, 26V-2003, P. Bariego, PB 2866.

Aunque ya había sido citada en la Sierra de la Cabrera (Nieto Feliner 1985: 171) y en el Macizo de Trevinca (Izco \& Ortiz 1985: 61), la subespecie es novedad para la flora vascular de la provincia de Zamora. Es una planta restringida a gleras y zonas rocosas de las cumbres, a diferencia de la subsp. hoffmanseguii (Samp.) G. Nieto que es más frecuente y se localiza preferentemente en las zonas bajas.

Doronicum carpetanum Boiss. \& Reuter ex Willk.

ESPAÑA: Zamora: Porto, Sierra de los Hoyos, 29TPG7874, $1650 \mathrm{~m}$, herbazales megaforbios, 12-VII-2003, P. Bariego, PB 3394; Galende, Laguna de la Roya, 29TPG8267, 1620 m, roquedo, 02-VII2002, P. Bariego, PB 2225; Porto, pr. embalse de Puente Porto, 29TPG8065, $1600 \mathrm{~m}$, brezal con carquesas, 27-VI-2002, P. Bariego \& A. Gastón, PB 1095; Cobreros, pr. Laguna de Sotillo, Vega del Teijo, 29TPG8362, 1610 m, canchal, 08-VIII-2002, P. Bariego, A. Gallego \& M. Santos, PB 2447; Galende, San Martín de Castañeda, Covadosos, 29TPG8671, $1640 \mathrm{~m}$, grietas en roquedos umbrosos, 11-VI-2002, P. Bariego, PB 1043.

Aportamos numerosas localidades a la previa de García López et al. (1992: 35), únicas conocidas de la provincia. Es una planta relativamente frecuente en herbazales megafórbicos, roquedos y canchales de las zonas elevadas de la Segundera.

\section{*Senecio pyrenaicus L.}

ESPAÑA: Zamora: Trefacio, pr. Laguna de Yeguas o Cubillas, 29TPG8774, $1840 \mathrm{~m}$, canchal, 20-VI-2002, P. Bariego \& A. Gallego, PB 1155; Porto, entre Riopedro y Vidulante, 29TPG8678, 1970 m, glera, 30-VII-2002, P. Bariego, PB 2406; Trefacio, pr. Laguna de la Yegüa, 29TPG8773, 1840 m, canchal, 29-VII-2002, P. Bariego, PB 2387. 
Novedad para la flora vascular de la provincia de Zamora, cuya localidad más cercana es de los Montes Aquilianos (Hergueta et al. 1999: 163). Es una planta relativamente frecuente en los canchales de las zonas elevadas de la Sierra Segundera.

\section{${ }^{*}$ Cirsium filipendulum Lange}

ESPAÑA: ZaMoRa: Porto, pr. núcleo urbano, 29TPG7370, 1230 $\mathrm{m}$, brezal-tojal, 31-VII-2003, P. Bariego, PB 3432.

Novedad para la Sierra Segundera y para la provincia de Zamora. Muy localizado entre matorrales, en zonas algo alteradas y con cierta nitrificación.

\section{Pilosella galliciana (Pau) Laínz}

ESPAÑA: ZAMORA: Porto, Valdecasares, 29TPG7961, $1850 \mathrm{~m}$, neveros, 08-VII-2003, P. Bariego, PB 3406; Porto, subida al Moncalvo, 29TPG8072, $1860 \mathrm{~m}$, matorral de aulaga sanabresa, 29-VI-2002, P. Bariego, PB 2212; Porto, pr. Laguna de Patos, hacia el Picón, 29TPG8578, $2010 \mathrm{~m}$, gleras finas de pizarras, 30-VII-2002, P. Bariego, PB 2403; Porto, pr. Moncalvo, 29TPG8175, $1980 \mathrm{~m}$, matorrales bajos con aulaga sanabresa, 25-VII-2002, P. Bariego, PB 800.

Aportamos nuevas localidades a la señalada previamente por Losa (1949: 139, sub. Hieracium auricula L.). Es una planta relativamente frecuente en los matorrales y pastizales psicroxerófilos de las zonas elevadas de la Sierra Segundera.

\section{Carex remota $\mathrm{L}$.}

ESPAÑa: Zamora: Galende, San Martín de Castañeda, Playa de la Sirena, 29TPG $8866,1000 \mathrm{~m}$, aliseda, 10-VIII-2002, P. Bariego, PB 875.

Novedad para la vertiente zamorana de la Sierra Segundera y segunda cita provincial (Navarro \& Valle 1983: 114). En esta zona es poco frecuente y únicamente se ha localizado en el entorno del Lago de Sanabria.

\section{Carex furva Webb}

ESPAÑA: ZAMORA: Trefacio, 29TPG8775,1980 m, naciente, 21VI-2002, P. Bariego \& A. Gallego, PB 2156; Porto, Moncalvo, 29TPG8074, $1980 \mathrm{~m}$, nevero, 27-VII-2002, P. Bariego \& E. Rico, PB 2363; Porto, Circo de Vidulante, 29TPG8479, $1880 \mathrm{~m}$, megaforbios en base de roquedos rezumantes, 14-VIII-2002, P. Bariego \& J. A. Sán- chez Agudo, PB 2466; Porto, Moncalvo, 29TPG8174, 2000 m, herbazales quionófilos en neveros en base de espolones rocosos, 27-VII2002, E. Rico \& P. Bariego, PB 841.

Nuevas localidades a añadir a las ya conocidas en este ámbito (Aedo et al., 1993: 366), únicas de esta planta en la provincia. En la Sierra Segundera es una planta muy localizada y restringida a algunos neveros de las zonas de cumbres.

\section{*Carex sylvatica Huds. subsp. sylvatica}

ESPAÑA: Zamora: Pías, Villanueva de la Sierra, Valle del Bibey, 29TPG6360, 1340 m, abedular, 20-VIII-2002, P. Bariego, PB 1886; Galende, Ribadelago, cañón del Segundera, 29TPG8464, 1370 $\mathrm{m}$, bordes de arroyo en acebeda, 08-VI-2002, P. Bariego, PB 1210; Galende, 29TPG8568, $1260 \mathrm{~m}$, prebosque ripícola, 25-VI-2002, P. Bariego, PB 2188.

Novedad provincial. Relativamente común en la vecina provincia de León (Molina et al. 2006: 35), no conocemos referencias hasta el momento del macizo galaico-leonés. En esta zona crece en herbazales ligados a diferentes tipos de bosques frescos y umbrosos.

\section{*Carex asturica Boiss.}

ESPAÑA: ZAMORA: Porto, Valle alto del Tera, 29TPG8278, 1700 $\mathrm{m}$, brezal de turbera, 19-VII-2002, P. Bariego, PB 2314; Porto, pr. Moncalvillo, 29TPG8073, $1990 \mathrm{~m}$, matorrales de brecina, 27-VI-2002, $P$. Bariego \& A. Gastón, PB 1108.

Salvo la indicación provincial en la monografía del género (Luceño 1994) y en Flora iberica (Luceño 2008), no conocemos citas concretas para Zamora, aunque si hay precedentes para el macizo de Trevinca (Nieto Feliner 1985: 195). Aparece salpicada por matorrales y pastos vivaces de la zona alta de la Sierra.

\section{*Poa chaixii Vill.}

ESPAÑA: ZamoRa: Porto, Las Tres Marías, 29TPG8275, 1850 $\mathrm{m}$, pastizal psicroxerófilo, 05-VIII-2002, P. Bariego \& M. Santos, PB 2431; Galende, San Martín de Castañeda, pr. Laguna Ventosa, 29TPG8572, 1800 m, cervunal, 02-VIII-2002, P. Bariego, PB 791.

Novedad provincial, aunque no para el macizo Galaico-Leonés (Nieto Feliner 1985: 189). En este territorio forma parte de pastizales psicroxerófilos y cervunales de las zonas elevadas. 


\section{Iris latifolia (Mill.) Voss}

ESPAÑA: ORENSE: Viana do Bolo, Pradorramisquedo, pr. embalse de San Agustín, 29TPG6667, 1100 m, roquedos rezumantes, 09-VII2003, P. Bariego, PB 3399; Zamora: Pías, Barjacoba, 29TPG6764, $1260 \mathrm{~m}$, repisas en roquedos, 09-VII-2003, P. Bariego, PB 3398; Galende, Cañón del Tera, 29TPG8568, $1200 \mathrm{~m}$, repisas en roquedos, 25 VI-2002, P. Bariego, PB 1185.

Nuevas localidades a añadir a la imprecisa de García López et al. (1992: 57). Es una planta poco frecuente que se restringe a algunos herbazales de orla forestal en enclaves soleados en repisas rocosas.

\section{*Dactylorhiza insularis (Sommier) Ó. Sánchez \& Herrero}

ESPAÑA: ZAMora: Galende, Ribadelago, Seoane, 29TPG8766, $1020 \mathrm{~m}$, bosque de ribera, P. Bariego, PB 3490, 15-V-2007.
Salvo la indicación provincial de Flora iberica (Sánchez Pedraja 2005) no conocemos otras referencias zamoranas de este taxon (Bariego \& Gastón 2005). Añadimos, por tanto, las primeras citas concretas para la Sierra Segundera y la provincia de Zamora. Únicamente se ha localizado una población en prados de siega próximos al Lago de Sanabria.

\section{AGRADECIMIENTOS}

Agradezco a María Santos, Álvaro Gallego, Aitor Gastón, Luis Delgado, José Ángel Sánchez Agudo y Enrique Rico la ayuda prestada en las jornadas de campo y en su asesoramiento científico. Los datos recogidos en este trabajo tienen su origen en su mayor parte en el trabajo de cartografía de hábitats del Parque Natural Lago de Sanabria y alrededores, financiado por la Junta de Castilla y León en el marco del proyecto "Cartografía detallada de hábitats del anexo I de la Directiva 92/43/CEE escala 1:10.000 en diversos espacios incluidos en la Red Natura 2000".

\section{BIBLIOGRAFÍA}

Aedo, C.; Aldasoro, J. J.; Argüelles, J. M.; Díaz Alonso, J. L.; González del Valle, J. M.; HerRá, C.; Laínz, M.; Moreno Moral, G.; Patallo, J. \& Sánchez Pedraja, O. 1993. Contribuciones al conocimiento de la flora cantábrica. Fontqueria 36: 349-374.

Aedo, C.; Aldasoro, J. J.; Argüelles, J. M.; Díaz Alonso, J. L.; Díez Rio, A.; González del Valle, J. M.; Laínz, M.; Moreno Moral, G.; Patallo, J. \& Sánchez Pedraja, 0. 1994. Contribuciones al conocimiento de la flora cantábrica, II. Fontqueria 40: 67-100.

Bariego Hernández, P.; Delgado Sánchez, L. \& Santos VICENTE, M. 2004. Aportaciones al conocimiento de la pteridoflora de la Cuenca del Duero (España). Bot. Complut. 28: 57-59.

Bariego, P. \& Gastón, A. 2005. Aproximación al catálogo de las orquídeas silvestres de la provincia de Zamora. Ecología 19: 149-164.

Cabezas Lefler, L.; Sevilla Felgendreher, P. \& Navarro AnDRÉS, F. 1987. Notas corológicas sobre plantas sanabresas, II. Stud. Bot. Univ. Salamanca 6: 95-98.

Carbó Nadal, R.; Mayor López, M.; Andrés Rodríguez, J. \& Losa QuiNTANA, J. M. 1977. Aportaciones al catálogo florfstico de la provincia de León. II. Acta Bot. Malac. 3: 63 - 120

Casaseca, B.; Giraldez, X.; Guillén, A. \& Rico, E. 1990. Manifestaciones eurosiberianas en la Sierra Segundera, Zamora (prolongación del eje Pirenaico-Cantábrico). Monogr. Inst. Piren. Ecol. Jaca 5: 111-120.

Castroviejo, S. \& Velayos, M. 1997. Sedum L. En: S. Castroviejo, C. Aedo, M. Laínz, R. Morales, F. Muñoz Garmen- dia, G. Nieto Feliner \& J. Paiva (Eds.), Flora iberica 5: $121-$ 153. Real Jardín Botánico, CSIC, Madrid.

CooK, C. D. K.; Grau, J. \& López González, G. 1986. Ranunculus L. En: S. Castroviejo, M. Laínz, G. López González, P. Montserrat, F. Muñoz Garmendia, J. Paiva \& L. Villar, L. (Eds.), Flora iberica 1: 279-371. Real Jardín Botánico, CSIC, Madrid.

García López, P.; Lauzurica, P.; Rey Quiroga, P. \& Roa MeDINA, A. 1992. Informe botánico del parque natural del Lago de Sanabria y sus alrededores. Junta Castilla y León. Valladolid.

García Río, R. \& Navarro Andrés, F. 1991. Comentarios sobre algunas cormófitas zamoranas. Stud. Bot. Univ. Salamanca 9: 149-152

García Río, R. \& NavarRo AndRÉs, F. 1994. Flora y vegetación cormofíticas de las comarcas zamoranas del Pan, Tera y Carballeda. Stvd. Bot. Univ. Salamanca 12: 23-202.

GómEz, D. 1997. Selinum L. En: G. Nieto Feliner, A. Herrero \& S. L. Jury (Eds.), Flora iberica 10: 308-312. Real Jardín Botánico, CSIC, Madrid.

Gómez Vigide, F.; García Martínez, X. R.; Pino Pérez, R.; González Domínguez, J.; Blanco-Dios, J. B.; CaAmaño Pórtela, J. L.; Pino Pérez, J. J.; Silva-Pando, F. J. \& VÁzQuez Míguez, A. C. 2005. Aportaciones a la flora de Galicia, VII. Nova Acta Ci. Compostelana Biol. 14: 57-68.

Gómez Vigide, F.; García Martínez, X. R.; Silva-Pando, F. J.; González Domínguez, J.; Blanco-Dios, J. B.; Rodríguez González, A.; Rial Pousa, S.; Álvarez Graña, D.; Caamaño Pórtela, J. L.; Pino Pérez, J. J. \& Pino Pérez, 
R. 2006. Aportaciones a la flora de Galicia, VIII. Nova Acta Ci. Compostelana Biol. 15: 53-63.

Gómez-Serrano, M. A. \& Mayoral, 0. 2006. Distribución y ecología de Rubus saxatilis L. (Rosaceae) en el Sistema Ibérico. Fl. Montib. 33: 32-36.

Hergueta, A. R.; Gamallo, G. L. \& Orduña, J. 1999. Aportaciones a la flora de los Montes Aquilianos (noroeste de la Península Ibérica). Nova Acta Ci. Compostelana (Bioloxia) 9: $159-164$

IzCo, J. \& ORTIZ, S. 1985. La vegetación glerícola del Macizo de Peña Trevinca. Lazaroa 7: 55-65.

LAínZ, M. 1967. Aportaciones al conocimiento de la flora gallega, V. Anal. Inst. Forestal Experiencias 12: 1-51.

Losa EspaÑa, T. M. 1949. Contribución al estudio de la flora de la provincia de Zamora. Plantas de Sierra Segundera, La Puebla de Sanabria, Calabor, etc. Inst. A. J. Cavanilles, secc. Barcelona. CSIC, Barcelona.

Losa EsPaña, T. M. 1950. Contribución al estudio de la flora de la provincia de Zamora. Contribución al estudio de la flora de la provincia de Zamora. (Addenda et corrigenda). Anales Jard. Bot. Madrid 9: 485-502.

LOSA EspaÑa, T. M. 1948. Algo sobre especies españolas del género «Euphorbia» L. Anales Jard. Bot. Madrid 7: 357-431.

LuceÑo, M. 1994. Monografía del género Carex en la Península Ibérica y Baleares. Ruizia 14: 1-139.

LuCEÑo, M. 2008. Carex L. En: S. Castroviejo, M. Luceño, A. Galán, P. Jiménez Mejías, F. Cabezas \& L. Medina (Eds.), Flora iberica 18: 109-250. Real Jardín Botánico, CSIC, Madrid.

Martín Bravo, S. \& LuceÑo, M. 2007. Notas corológicas de resedáceas para la Península Ibérica. Acta Bot. Malac. 32: 221-227.
Molina, A.; AcEDo, C. \& Llamas, F. 2006. Observaciones sobre el género Carex en la provincia de León (NW España). Lagascalia 26: 25-37

NavarRo Andrés, F. \& Valle GutiérRez, C. J. 1983. Fitocenosis fruticosas de las comarcas zamoranas de Tábara, Alba y Aliste. Stvd. Bot. Univ. Salamanca 2: 69-121.

Nieto Feliner, G. 1985. Estudio crítico de la flora orófila del suroeste de León: Montes Aquilianos, Sierra del Teleno y Sierra de la Cabrera. Ruizia 2: 3-239.

ORTIZ, S. 1986a. Apuntes sobre la flora ourensana. Stvd. Bot. Univ. Salamanca 5: 191-194.

ORTIZ, S. 1986b. Series de vegetación y su zonación altitudinal en el macizo de Pena Trevinca y Sierra do Eixo. Tesis doctoral inédita. Universidad de Santiago de Compostela.

Pulgar, I.; Iglesias, R. \& SoÑoRA, X. 1998. Apuntamentos sobre a flora vascular galega, XVI. Nova Acta Ci. Compostelana (Bioloxía) 8: 91-94.

Roselló, J.A. 1997. Sempervivum L. En: S. Castroviejo, C. Aedo, M. Laínz, R. Morales, F. Muñoz Garmendia, G. Nieto Feliner \& J. Paiva (Eds.), Flora iberica 5: 110-116. Real Jardín Botánico, CSIC, Madrid.

SÁnchez PedrajA, 0. 2005. Dactylorhiza Neck. ex Nevski En: C. Aedo \& A. Herrero (Eds.), Flora iberica 21: 94-111. Real Jardín Botánico de Madrid. CSIC.

SÁncheZ RodrígueZ, J. A. 1986. Aportaciones a la flora zamorana, I. Collect. Bot. (Barcelona) 16: 371-376.

Silva Pando, F. J. 2008. Las plantas endémicas y subendémicas de Galicia. Bol. BIGA 3: 9-150.

VALDÉs, B. 2008. Notas sobre el género Myosotis (Boraginaceae). Lagascalia 28: 103-116.

Vitek, E. 2009. Euphrasia L. En: C. Benedí, E. Rico, J. Güemes \& A. Herrero (Eds.), Flora iberica 13: 454-473. Real Jardín Botánico, CSIC, Madrid. 